\title{
Cadmium stress tolerance in plants: a key role of endogenous and exogenous salicylic acid
}

\author{
Aïcha Belkadhi*, Wahbi Djebali, Hédia Hédiji, Wided Chaïbi \\ Faculty of Sciences of Tunis, Physiology and Biochemistry of Plant Response to Abiotic Stresses Unit, University of Tunis El Manar, \\ 1060, Tunis El Manar, Tunisia
}

\author{
Article history \\ Received: 5 January 2016 \\ Accepted: 14 February 2016 \\ Published: 18 February 2016 \\ CC Belkadhi et al. (2016) \\ Editor \\ K. K. Sabu \\ Publisher \\ Horizon e-Publishing Group \\ Corresponding Author \\ Aïcha Belkadhi \\ \aicha585@yahoo.ca
}

\begin{abstract}
Cadmium (Cd) has become one of the major metal stresses which pose a serious threat to plants and animals. In this context, endogenous and exogenous salicylic acid (SA) could play an important role in mitigating the uptake of the $\mathrm{Cd}$ ions and providing immunity to plants against the heavy metal stress. SA enhances the resistance capacity of contaminated plants, which, however, depends on the metal concentration and the duration of the treatment. Moreover, SA is considered as a promising signal molecule for improving the efficiency of phytoremediation, and, consequently, growing of safe crops in metal polluted areas. The recent developments in the probable mechanisms by which SA could enhance the tolerance of plants to heavy metals and how it could have an effect on phytoremediation of $\mathrm{Cd}$ from contaminated soils are discussed.
\end{abstract}

Keywords

Cadmium stress; endogenous salicylic acid; exogenous elicitor; phytoremediation

Belkadhi, A., Djebali, W., Hédiji, H., and Chaïbi, W. 2016. Cadmium stress tolerance in plants: a key role of endogenous and exogenous salicylic acid. Plant Science Today 3(1): 48-54. http://dx.doi.org/10.14719/pst.2016.3.1.181

\section{Introduction}

Cadmium (Cd) is generally known as the most toxic pollutants in the environment. Furthermore, this heavy metal has a high mobility in soil and is easily absorbed by plant roots (Belkadhi et al., 2015 a). Exogenous application of salicylic acid (SA) on the other hand is able to leach and immobilize $\mathrm{Cd}$ in soils (Guo et al., 2007). The enhancement of tolerance to Cd stress in SA-treated plants is welldocumented (Choudhury and Panda, 2004; Zhang et al., 2011; Guo et al., 2007, 2013; Li et al., 2014; Belkadhi et al., 2015 a, b). For this, SA induces genes that are responsible for resistance to $\mathrm{Cd}$ and have evolved a variety of mechanisms to reduce the stress (Shi and Zhu, 2008). These mechanisms include the complex formation and sequestration of Cd (Metwally et al., 2003; Belkadhi et al., 2012), reduction of metal to less toxic forms, generation of the oxidative stress response, reduced membrane permeability, and a direct removal of the metal (Choudhury and Panda, 2004; Guo et al., 2007; Li et al., 2014; $\mathrm{Xu}$ et al., 2015). There is also evidence which suggests that SA is capable of facilitating plant growth and reducing/detoxifying Cd toxicity and could be a promising tool in increasing the phytoremediation efficiency (Singer et al., 2003). Considering this as a basis, Tao et al. (2013) studied the role of endogenous SA in plant response to lead $(\mathrm{Pb})$ or $\mathrm{Cd}$ by the means of wild-type Arabidopsis and its SA producing mutant snc1, SA-reducing transgenic line nahG, SA signal-blocking npr1-1, as well as expression of nahG in snc1 plant (snc1/nahG) with a comparable level of SA to the wild-type. The results showed that $\mathrm{Pb}$ - or $\mathrm{Cd}$ induced phytotoxicity in Arabidopsis was intensified by elevated endogenous SA, whereas ameliorated by reduced SA. 
Analogously to biotic stress, SA also induces a systemic acquired resistance (SAR) in plants exposed to $\mathrm{Cd}$ via SA-dependent signal transduction pathway (Volt et al., 2009). In addition, $\mathrm{Cd}$ bioaccumulation induces the synthesis of SA, hydrogen peroxide $\left(\mathrm{H}_{2} \mathrm{O}_{2}\right)$, and other essential metabolites that finally cause SAR and the resistance to metal stress (Kovács et al., 2014). In this context, the role of SA in plant defense against $\mathrm{Cd}$ stress should be considered. In spite of scarce information on the defensive mechanisms by plants via endogenous production of SA, it is known that exogenous SA application may have the potential to activate inducible plant defense systems (Guo et al., 2007, 2013; Li et al., 2014). There are different ways by which SA application can alleviate the $\mathrm{Cd}$ toxicity caused to plants.

For example, presoaking of seeds with SA, reduces the availability and mobility of the metal by enhancing its chelation and detoxification via forming a complex with SH-groups (Belkadhi et al., 2012), its binding with siderophores (Sinha and Mukherjee, 2008), and enhancement of the antioxidant capacity (Radwan, 2012; Belkadhi et al., 2014). SA also reduces indirectly the impact of $\mathrm{Cd}$ by the secretion of biologically active substances, such as other plant growth-stimulating hormones (Tamás et al., 2012; Shakirova et al., 2016). The beneficial effects on the plant growth in the presence of $\mathrm{Cd}$ have been attributed to SA and may include an osmotic adjustment and stomatal regulation (Krantev et al., 2008), modification of organ morphology (Belkadhi et al., 2013), enhanced uptake of minerals (Drazic et al., 2006), and the alteration of the nitrogen accumulation and metabolism (Koç et al., 2013).

\section{Indirect reduction of cadmium toxicity by salicylic acid}

SA could contribute to the plant resistance to $\mathrm{Cd}$ stress indirectly by increasing the overall fertility of the contaminated soil and by supplementing nutrients, such as, $\mathrm{P}, \mathrm{Mg}, \mathrm{Ca}$ and $\mathrm{Fe}$ to stressed plants (Drazic et al., 2006). As a result of this activity, the growth and health of plants are improved. One of the mechanisms of the plant growth promotion by SA is the synthesis of growth-promoting regulator (Tamás et al., 2014; Shakirova et al., 2016). Besides, the exogenous application of SA facilitated the plants in maintaining their improved growth (Shakirova et al., 2016). Recently, Guan et al. (2015) established that a glutathione synthetase (GSHS)-like gene from Lycium chinense maybe regulated by $\mathrm{Cd}$ induced endogenous SA. In fact, glutathione accumulation occurred via enhanced LcGSHS gene expression and the SA signaling cascade was implicated in this accumulation. Additionally, the overexpression of LcGSHS in transgenic Arabidopsis resulted in improvement of tolerance to $\mathrm{Cd}$ stress than wild-types. Pre-treatment of wheat seeds with SA has been reported to ameliorate the effects of Cd-induced heavy metal toxicity via enhanced activities of reactive oxygen species (ROS)-scavenging enzymes (Agami and Mohamed, 2013). Although the authors did not analyze the photosynthetic activities, improved growth parameters were estimated to be related to the improved contents of photosynthetic pigments such as chlorophyll a, b and carotenoids in Cdtreated plants. However, the beneficial effect of SA on leaf structure may be due to the crucial role in cell division and expansion.

In order to enlighten the potential mechanisms underlying SA-mediated improved $\mathrm{Cd}$ stress tolerance in plants, this section assesses in a consice manner current reports available on the SA-involvement in ROS-signaling and the modulation of defense responses. Both endogenous and exogenous SA were evidenced to play roles in plant metabolism during defense responses (Kang et al., 2014; Khan et al., 2012; 2015). Moreover, the synchronization of dependent and independent SA-signaling components with ROS-signaling provided an appropriate defense response (Khan et al., 2015). SA can act as a signal for the development of the SAR (Shirasu et al., 1997), and can also induce the activation of a protein kinase (Mikolajczyk et al., 2000). Arabidopsis thaliana plants were able to recognize the response of ROS-SA interaction via an opposed action of SA and SA-signaling on apoplastic ROS-signaling (Xu and Brosché, 2014). Furthermore, activation of the SA signaling pathway was involved in the responses to $\mathrm{Cd}$ rhizotoxicities in $A$. thaliana, explaining the involvement of this signaling pathway in the tolerance mechanisms (Zhao et al., 2009).

\section{Salicylic acid: Protection against cadmium- induced oxidative stress}

The exposure of plants to $\mathrm{Cd}$ results in an oxidative stress as showed by protein carbonylation (Djebali et al., 2008), lipid peroxidation (Guo et al., 2007), and ROS production (Djebali et al., 2005; Rodríguez-Serrano et al., 2006; Zhang et al., 2011; Zhang and Chen, 2011; Tamás et al., 2015). Cd could also incite a depletion of glutathione and an inhibition of antioxidative enzymes (Jin et al., 2008). The improvement of the plant ROS-detoxification system by SA can be considered as a promising approach to protect plants from the poisonous effects of Cd stress (Rodríguez-Serrano et al., 2006, Zhang et al., 2011, Belkadhi et al., 2015; Tamás et al., 2015). It is reported that SA is a monophenol that acts as an antioxidant and could complement the deficient antioxidative systems of the plants (Garib and Hegazi, 2010). Both exogenous and endogenous SA mitigate much kind of ROS including hydrogen peroxide $\left(\mathrm{H}_{2} \mathrm{O}_{2}\right)$, superoxide anion $\left(\mathrm{O}_{2}^{-}\right)$, hydroxyl radicals $(\cdot \mathrm{OH})$, singlet oxygen $\left({ }^{1} \mathrm{O}_{2}\right)$ (Shah and Klessig, 1999). According to Panda 
and Patra (2007), in Cd stress conditions, SAtreated plants could be protected by maximal stimulation of antioxidant molecules and enzymes such as ascorbate, guaiacol peroxidase (GPX, EC 1.11.1.7), and superoxide dismutase (SOD, EC 1.15.1.1), as compared to untreated plants, indicating stimulation of plant immunity by SA (Yang and Dong, 2014).

Moreover, Sappl et al. (2004) approved 20 gluthatione-S-transferase (GST, EC 2.5.1.13) isoforms in Arabidopsis cell culture responding to SA treatment with a combination of GST antibody detection, LC-MS/MS analysis of 23-30 kDa proteins and glutathione-affinity chromatography. A decrease in the content of non-protein thiols (NP-SHs) in shoots was likely to be associated with the improved antioxidant enzymes activities in roots due to low metal transport in the aboveground parts of the plant (Belkadhi et al., 2012; 2013). On the other hand, the exogenous application of SA stimulates both the accumulation and production of phenolic compounds by plants, known as antioxidants and chelators of $\mathrm{Cd}$, particularly in the plant roots (Kovácik et al., 2009). Besides, Kovács et al. (2014) showed that exposure to Cd induced SA synthesis, especially in the leaves, and that the phenylpropanoid synthesis pathway is accountable for the SA accumulation observed after $\mathrm{Cd}$ stress. Although no direct relationship was observed between the primary SA levels and the degree of Cd tolerance, the results suggested that the increase of SA levels in roots of wheat varieties during Cd stress could be related with the enhancement of the inner glutathione cycle, therefore endorsing the antioxidant and metal detoxification systems, which improve $\mathrm{Cd}$ stress tolerance in wheat plants. The direct relationship between some SAassociated compounds and protective compounds suggested that SA-related signaling may also play a role in the plant adaptation to the heavy metal stress.

\section{Regulation of the antioxidant metabolism under cadmium stress by salicylic acid}

SA-pretreatment was well proven to alleviate the deleterious effects of Cd stress in Triticum aestivum (Shakirova et al., 2016) and in Oryza sativa and Cucumis melo through enhancing the activities of antioxidant enzymes including SOD, CAT, GPX, APX, and GR (Guo et al., 2007; Zhang et al., 2015). Activities of $\mathrm{H}_{2} \mathrm{O}_{2}$-metabolizing enzymes (such as CAT, POD, and APX) and SOD enzymes were also modulated with exogenous SA in flax roots and leaves exposed to Cd (Belkadhi et al., 2013; Belkadhi et al., 2014). Restriction of Cduptake in presence of SA $(50 \mu \mathrm{M})$ suggests an integration of different signals and SA-enhanced Cd tolerance due to SA-regulated Cd uptake as well as the SA-elevated enzymatic and non-enzymatic antioxidant pool in rice (Singh and Shah, 2015). Ascorbate and glutathione, as redox active compounds have been extensively reported to keep up a homeostatic balance of the cellular redox status, and are involved in protective mechanisms against Cd stress (Anjum et al., 2014, 2015; Noctor et al., 2012; Khan et al., 2015). In Cdexposed T. aesticum varieties, SA-signaling was connected to glutathione-related mechanisms (Kovács et al., 2014). SA-mediated differential regulation of the transcript levels of the gene encoding GSH synthetase (GSHS) enzyme was advocated as a major mechanisms underlying previous role of endogenous SA in Cd tolerance via the regulation of LcGSHS transcript expression levels (Guan et al., 2015).

Although numerous reports showed that SA exogenous application can alleviate oxidative damage caused by $\mathrm{Cd}$ on different plant species, the results presented by Zawoznik et al. (2007) indicated that endogenous SA acted as a potentiating mediator of the oxidative stress triggered by the exposure to $\mathrm{Cd}$ in Arabidopsis plants. Transgenic plants expressing the bacterial salicylate hydroxylase gene and as a consequence unable to synthesize SA confirmed no data of being affected by $\mathrm{Cd}$ exposure after 5 days of $\mathrm{Cd}$ treatment, while wild type plants did. SA $(500 \mu \mathrm{M})$ improved Cd-tolerance and photosynthetic capacity in hemp (Cannabis sativa) by increasing both SOD and POD activities (Shi et al., 2009). In another instance the applied SA-induced SOD activity accompanied an enhancement of $\mathrm{Ca}^{2+}$ (a second messenger) in shoots and incited a transient increase in $\mathrm{H}_{2} \mathrm{O}_{2}$ which in turn was argued to stimulate the activities of many antioxidant enzymes and eventually to diminution in intracellular ROS amounts (Hara et al., 2012). Recently, in Medicago sativa, a tight link between Haem oxygenase (EC 1.14.99.3), HO-1-mediated and SA-dependent signaling existed in the alleviation of Cd toxic effects; SA-mediated HO-1 up-regulation was implicated in the induction of antioxidative behavior in the root tissues (Cui et al., 2012). On the other hand, Metwally et al. (2003) measured the transcript levels of six genes related to antioxidant defense by semi-quantitative reverse transcriptase (RT)-PCR, which showed the suppression of Cd-induced up-regulation of transcript amounts in the SA presoaked barley samples, however, the transcript of glutathione synthase (GS, EC 6.3.2.3) that was already existent at elevated amounts in the SA-presoaked control but down-regulated in the presence of $\mathrm{Cd}$ in the nutrient solution.

\section{Salicylic acid: Importance in phytoremediation technologies}

Integration of plant defense activators is a novel approach to development of an integrated strategy in order to phytoremediate $\mathrm{Cd}$ contamination in soils that require to be cleaned up. SA in this context may exert positive effects on plants through various mechanisms and is reported to 
enhance phytoremediation strategy. In fact, SA has been shown to enhance phytoremediation efficiency (Tao et al., 2013). Although, no study has showed that endogenous or exogenous SA alone may improve $\mathrm{Cd}$ bioavailability and accumulation (Drazic and Mihailovic, 2005), a comparison between the dye contaminated soil and remediated soil revealed that SA decreased in the leaves in the dye contaminated soil than in remediated soil (Jayanthy et al., 2014). The results also exhibited that the level of SA decreased significantly before remediation and enhanced after remediation in both Vigna radiata roots and leaves. Regarding the latter, increasing evidence has demonstrated that exposure to $\mathrm{Cd}$ increases endogenous SA contents, and the exogenous application of SA can improve the tolerance of plants to heavy metals, demonstrating that SA is implicated in the responses of plants to the heavy metal stress (Metwally et al., 2003; Horváth et al., 2007; Pál et al., 2006). In addition, Tao et al. (2013) showed that high endogenous SA intensified Cdinduced phytotoxicity, while low concentration of SA ameliorated the toxicity, which was further proven by an unclaimed performance of double mutant snc1/nahG plants relative to the snc1 plants with high SA content. However, due to the significant difference of initial levels of endogenous SA in a wide range of plant species (Rivas-San Vicente and Plasencia, 2011); it will eventually be tricky to assess precisely which role was played by SA in response of plants to the heavy metal stress by means of an exogenous application (Gururani et al., 2015).

In parallel, most of the SA-applying studies demonstrated that treatments with SA can enhance $\mathrm{Cd}$ tolerance in a wide range of plant species (Guo et al., 2007; Horváth et al., 2007; Pál et al., 2006; Belkadhi et al., $2015 \mathrm{a}, \mathrm{b}$ ), which means that this signal molecule could play an important role in $\mathrm{Cd}$ sequestration and chelation (Metwally et al., 2003). In this context, Hao et al. (2012) studied the effect of SA, swine manure, and potassium chloride $(\mathrm{KCl})$ applications, on the growth, uptake and translocation of $\mathrm{Cd}$ and zinc (Zn) of Helianthus annuus grown on a contaminated soil. The results showed that SA reduced the $\mathrm{Cd} / \mathrm{Zn}$ ratios in flower of sunflower, while $\mathrm{KCl}$ significantly increased the $\mathrm{Cd} / \mathrm{Zn}$ ratios. Large body of evidence has positively confirmed that both endogenous and exogenous SA are crucial in plant responses toward heavy metal stresses, but their roles in phytoremediation are still ambiguous and further studies are required. Providentially, the method of reverse genetics has been widely facilitated to analyze the SA-related mechanisms, and a number of Arabidopsis mutants with gain-of-function or loss-of-function in SA-dependent signaling have been identified (Nawrath et al., 2002; Lu et al., 2003). Although A. thaliana is not a hyperaccumulator, it represents an appropriate material used to study metal accumulation and acclimation mechanisms (Halimaa et al., 2014).

\section{Conclusion}

Both endogenous and exogenous SA play an imperative role in plant tolerance against $\mathrm{Cd}$ stress. Endogenous SA induces plant defense against $\mathrm{Cd}$ exposure through various physiological, biochemical and molecular mechanisms. Biochemical basis of SA-induced defense has been established to be very dynamic with profound effect on the stress, thereby allowing the plants to withstand it. Additionally, exogenous SA has been proved advisable in enhancing resistance to $\mathrm{Cd}$ stress in many plant species. Another important perspective is its plausible agricultural use for improving crop yield. In addition, SA use in phytoremediation is a new study in the field of plant physiology. Seed priming with SA is the most pragmatic approach for agricultural purposes. An important question of this study was how exogenous SA ensured protection against $\mathrm{Cd}$ induced oxidative stress and regulation of the antioxidant metabolism. Alternatively, the positive effects of SA were related to the expression of specific genes coding for defense-related proteins or enzymes. These results may afford good frameworks for strategies aimed at manipulating plants for increasing $\mathrm{Cd}$ content in order to develop crops capable for removing efficiently the heavy metal contaminants from the soils.

\section{Competing Interests}

The authors declare that they have no competing interests.

\section{Acknowledgements}

We wish to thank authors who contributed to the study exemplified in this article.

\section{References}

Agami, R.A., and G.F. Mohamed. 2013. Exogenous treatment with indole-3-acetic acid and salicylic acid alleviates cadmium toxicity in wheat seedlings. Ecotoxicology and. Environmental Safety 94:164-171. doi: 10.1016/j.ecoenv.2013.04.013. PMID: 23684274.

Anjum, N. A., S.S. Gill, R. Gill, M. Hasanuzzaman, A.C. Duarte, E. Pereira, I. Ahmad, R. Tuteja R, and Tuteja N. 2014. Metal/metalloid stress tolerance in plants: role of ascorbate, its redox couple and associated enzymes. Protoplasma 251: 1265-1283. doi: 10.1007/s00709-014-0636-x. PMID: 24682425.

Anjum N.A., Umar S., Aref I.M., and Iqbal M. 2015. Managing the pools of cellular redox buffers and the control of oxidative stress during the ontogeny of drought-exposed mungbean (Vigna radiata L.)-role of sulfur nutrition. Frontiers in Environmental Science 2:66. doi: 10.3389/fenvs.2014.00066.

Belkadhi, A., H. Hediji, Z. Abbes, W. Djebali, and W. Chaïbi. 2012. Influence of salicylic acid 
pretreatment on cadmium tolerance and its relationship with non-protein thiol production in flax root. African Journal of Biotechnology 11: 9788-9796. doi: 10.5897/AJB11.2051.

Belkadhi, A., A. De Haro, P. Soengas, S. Obregón, M. E. Cartea, W. Djebali, W. Chaïbi. 2013. Salicylic acid improves root antioxidant defense system and total antioxidant capacities of flax subjected to cadmium. OMICS: A Journal of Integrative Biology 17(7): 398-406. doi: 10.1089/omi.2013.0030. PMID: 23758477.

Belkadhi, A., A. De Haro, P. Soengas, S. Obregón, M. E. Cartea, W. Chaïbi, and W. Djebali. 2014. Salicylic acid increases tolerance to oxidative stress induced by hydrogen peroxide accumulation in leaves of cadmium-exposed flax (Linum usitatissimum L.). Journal of Plant Interactions 9(1): 647-654. doi: 10.1080/17429145.2014.890751.

Belkadhi A., A. De Haro, S. Obregon, W. Chä̈bi, W. Djebali. 2015. Positive effects of salicylic acid pretreatment on the composition of flax plastidial membrane lipids under cadmium stress. Environmental Science and Pollution Research 22 (2): 1457-1467. doi: 10.1007/s11356014-3475-6. PMID: 25163565.

Belkadhi A., A. De Haro, S. Obregon, W. Chaübi, and W. Djebali. 2015. Exogenous salicylic acid protects phospholipids against cadmium stress in flax (Linum usitatissimum L.). Ecotoxicology and Environmental Safety 120: 102-109. doi: 10.1016/j.ecoenv.2015.05.028. PMID: 26057076.

Belkhadi, A., H. Hediji, Z. Abbes, I. Nouairi, Z. Barhoumi, M. Zarrouk, W. Chaïbi, and W. Djebali. 2010. Effects of exogenous salicylic acid pre-treatment on cadmium toxicity and leaf lipid content in Linum usitatissimum L. Ecotoxicology and Environmental Safety 73: 1004-1011. doi: 10.1016/j.ecoenv.2015.05.028. PMID: 26057076.

Choudhury, S., and S.K. Panda. 2004. Role of salicylic acid in regulating cadmium induced oxidative stress in Oryza sativa L. roots. Bulgarian Journal of Plant Physiology 30: 95-110.

Cui, W., L. Li, Z.Z. Gao, H.H. Wu, Y. Xie, and W. Shen. 2012. Haem oxygenase-1 is involved in salicylic acid-induced alleviation of oxidative stress due to cadmium stress in Medicago sativa. Journal of Experimental Botany 63: 5521-5534. doi: 10.1093/jxb/ers201. PMID: 22915740.

Djebali , W., M. Zarrouk, R. Brouquisse, S. El Kahoui, F. Liman, M.H. Ghorbel, and W. Chaïbi. 2005. Ultrastructure and lipid alterations induced by cadmium in tomato Lycopersicon esculentum chloroplast membranes. Plant Biology 7: 258-368. doi: 10.1055/s-2005-837696. PMID: 16025408.

Djebali, W., P. Gallusci, C. Polge, L. Boulila, N. Galtier, P. Raymond, W. Chaibi, and R. Brouquisse. 2008. Modifications in endopeptidase and $20 \mathrm{~S}$ proteasome expression and activities in cadmium treated tomato Solanum lycopersicum L. plants. Planta 227: 625-639. doi: 10.1007/s00425-007-06446. PMID: 17952456.

Drazic, G., and N. Mihailovic. 2005. Modification of cadmium toxicity in soybean seedlings by salicylic acid. Plant science 168: 511-517. doi: 10.1104/pp.102.018457. PMID: 166972.
Drazic, G., N. Mihailovic, and M. Lojic. 2006. Cadmium accumulation in Medicago sativa seedlings treated with salicylic acid. Biologia Plantarum 50: 239-244. doi: 10.1007/s10535-006-0013-5.

Gharib, F.A., A.Z. Hegazi. 2010. Salicylic acid ameliorates germination, seedling growth, phytohormone and enzymes activity in bean (Phaseolus vulgaris L.) under cold stress. Journal of American Science 6(10): 675-683. doi: 10.3389/fpls.2015.00462. PMID: 4485163.

Guan, C., J. Ji, C. Jia, W. Guan, X. Li, C. Jin, and G. Wang. 2015. A GSHS-like gene from Lycium chinense maybe regulated by cadmium induced endogenous salicylic acid and overexpression of this gene enhances tolerance to cadmium stress in Arabidopsis. Plant Cell Reports 34: 871-884. doi: 10.1007/s00299-015-1750-8. PMID: 25627256.

Guo, B., Y.C. Liang, Y.G. Zhu, and F.J. Zhao. 2007. Role of salicylic acid in alleviating oxidative damage in rice roots (Oryza sativa) subjected to cadmium stress. Environmental Pollution 147: 743-749. doi:10.1016/j.envpol.2006.09.007. PMID: 17084493.

Guo, Q., L. Meng, P.C. Mao, Y.Q. Jia, and Y.J. Shi. 2013. Role of exogenous salicylic acid in alleviating cadmium-induced toxicity in Kentucky bluegrass. Biochemical Systematics and Ecology 50: 269-276. doi:10.1016/j.bse.2013.05.002.

Gururani, M.A., J. Venkatesh, M. Ganesan, R.J. Strasser, Y. Han, J.I. Kim, H.I. Lee, and P.S. Song. 2015. In vivo assessment of cold tolerance through chlorophyll-a fluorescence in transgenic zoysiagrass expressing mutant phytochrome A. PLOS ONE 10: e0127200. doi: 10.1371/journal.pone.0127200. PMID: 4444231.

Halimaa, P., D. Blande, M.G. Aarts, M. Tuomainen, A. Tervahauta, and S. Kärenlampi. 2014. Comparative transcriptome analysis of the metal hyperaccumulator Noccaea caerulescens. Frontiers in Plant Science 5: 213. doi: 10.3389/fpls.2014.00213. PMID: 4033236.

Hara, M., J. Furukawa, A. Sato, T. Mizoguchi, and K. Miura. 2012. Abiotic stress and role of salicylic acid in plants. In: Ahmad P, Parsad MNV (eds) Abiotic stress responses in plants: metabolism, productivity and sustainability. Springer Science, New York, 235-251. doi: 10.1007/978-1-4614-06341_13.

Horvath, E., G. Szalai, and T. Janda. 2007. Induction of abiotic stress tolerance by salicylic acid signaling. Journal of Plant Growth Regulation 26: 290-300. doi: 10.3389/fpls.2015.00462. PMID: 4485163.

Jayanthy, V., R. Geetha, R. Rajendran, P. Prabhavathi, S. Karthik Sundaram, S. Dinesh Kumar, and P. Santhanam. 2014. Phytoremediation of dye contaminated soil by Leucaena leucocephala (subabul) seed and growth assessment of Vigna radiata in the remediated soil. Saudi Journal Biological Sciences 21(4): 324-333. doi: 10.1016/j.sjbs.2013.12.001. PMID: 25183943.

Jin, X., X. Yang, E. Islam, D. Liu, and Q. Mahmood. 2008. Effects of cadmium on ultrastructure and antioxidative defense system in hyperaccumulator and non-hyperaccumulator ecotypes of Sedum alfredii Hance. Journal of 
Hazardous Materials 156 (1-3): 387-397. doi: 10.1016/j.jhazmat.2007.12.064. PMID: 18242844.

Kang, G., G. Li, and T. Guo. 2014. Molecular mechanism of salicylic acid-induced abiotic stress tolerance in higher plants. Acta Physiologiae Plantarum 36: 2287-2297. doi: 10.1007/s11738-014-1603-z.

Khan, N. A., R. Nazar, N. Iqbal, and N.A. Anjum. 2012. Phytohormones and Abiotic Stress Tolerance in Plants. Berlin: Springer. doi: 10.1007/978-3-64225829-9.

Khan, M.I.R., M. Fatma, T.S. Per, N.A. Anjum, and N.A. Khan. 2015. Salicylic acid-induced abiotic stress tolerance and underlying mechanisms in plants. Frontiers in Plant Science 6: 462. doi: 10.3389/fpls.2015.00462. PMID: 4485163.

Koç, E., Üstün, A.S., and N. Çelik. 2013. Effect of exogenously applied salicylic acid on cadmium chloride-induced oxidative stress and nitrogen metabolism in tomato (Lycopersicon esculentum L.). Turkish Journal of Biology 37: 361-369. doi: 10.3906/biy-1211-13.

Kováčik, J., B. Klejdus, J. Hedbavny, and M. Bačkor. 2009. Salicylic acid alleviates NaCl-induced changes in the metabolism of Matricaria chamomilla plants. Ecotoxicology 18: 544-554. doi: 10.1007/s10646009-0312-7. PMID: 19381803.

Kovács, V., O.K. Gondor, G. Szalai, I. Majláth, T. Janda and M. Pál. 2014. UV-B radiation modifies the acclimation processes to drought or cadmium in wheat. Environmental and Experimental Botany 100:

122-131. doi:10.1016/j.envexpbot.2013.12.019.

Krantev, A., R. Yordanova, T. Janda, G. Szalai, L. and Popova. 2008. Treatment with salicylic acid decreases the effect of cadmium on photosynthesis in maize plants. Journal of Plant Physiology 165: 920-931. doi:10.1016/j.jplph.2006.11.014. PMID: 17913285.

Li, X., L. Ma, N. Bu, Y. Li, and L. Zhang. 2014. Effects of salicylic acid pre-treatment on cadmium and/or UV-B stress in soybean seedlings. Biologia Plantarum 58: 195-199. doi: 10.1007/s10535-0130375-4.

Lu, H., D.N. Rate, J.T. Song, and J.T. Greenberg. 2003. ACD6, a novel ankyrin protein, is a regulator and an effector of salicylic acid signaling in the Arabidopsis defense response. Plant Cell 15: 24082420. doi: 10.1105/tpc.015412. PMID: 14507999.

Metwally, A., I. Finkemeier, M. Georgi, and K.J. Dietz. 2003. Salicylic acid alleviates the cadmium toxicity in barley seedlings. Plant Physiology 132 : 272-281. doi: 10.1104/pp.102.018457. PMID: 12746532 .

Mikolajczyk, M., O.S. Awotunde, G. Muszynska, D.F. Klessig, and G. Dobrowolska. 2000. Osmotic stress induced rapid activation of a salicylic acidinduced protein kinase and a homolog of protein kinase ASK1 in tobacco cells. Plant Cell 12: 165178. doi: 10.1105/tpc.12.1.165. PMID: 149182.

Nawrath, C., S. Heck, N. Parinthawong, and J.P. Metraux. 2002. EDS5, an essential component of salicylic acid-dependent signaling for disease resistance in Arabidopsis, is a member of the MATE transporter family. Plant Cell 14:275-86. doi: 10.1105/tpc.010376. PMID: 150564.
Noctor, G., A. Mhamdi, S. Chaouch, Y. Han, J. Neukermans, B. Marquez-Garcia, G. Queval, and C.H. Foyer. 2012. Glutathione in plants: an integrated overview. Plant Cell and Environment 35: 454-484. doi: 10.1111/j.13653040.2011.02400.x. PMID: 21777251.

Pál, M., E. Horváth, T. Janda, E. Páldi, and G. Szalai. 2006. Physiological changes and defense mechanisms induced by cadmium stress in maize. Journal of Plant Nutrition and Soil Science 169: 239- 246. doi: 10.1002/jpln.200520573.

Panda, S.K., and Patra HK. 2007. Effect of salicylic acid potentiates cadmium-induced oxidative damage in Oryza sativa L. leaves. Acta Physiologiae Plantarum 29: 567-575. doi: 10.1007/s11738-0070069-7.

Radwan, D.E.M. 2012. Salicylic acid induced alleviation of oxidative stress caused by clethodim in maize (Zea mays L.) leaves. Pesticide Biochemistry and Physiology 102:

182-188. doi:10.1016/j.pestbp.2012.01.002.

Rivas-San Vicente, M., and J. Plasencia. 2011. Salicylic acid beyond defence: its role in plant growth and development. Journal of Experimental Botany 62: 3321-38. doi: 10.1093/jxb/err031. PMID: 21357767.

Rodriguez-Serrano, M., M.C. Romero-Puertas, G.M. Pastori, F.J. Corpas, L.M. Sandalio, L.A. Del Rio, and J.M. Palma. 2007. Peroxisomal membrane manganese superoxide dismutase: characterization of the isozyme from watermelon (Citrullus lanatus Schrad.) cotyledons. Journal of Experimental Botany 58: 2417-2427. doi: 10.1093/jxb/erm095. PMID: 17545229.

Sappl, P.G., L. Onate-Sanchez, K.B. Singh, and A.H. Millar. 2004. Proteomic analysis of glutathione $S$ transferases of Arabidopsis thaliana reveals differential salicylic acid-induced expression of the plant-specific phi and tau classes. Plant Molecular Biology 54: 205-219. doi: 10.1023/B:PLAN.0000028786.57439.b3. PMID: 15159623.

Shah, J., and D.F. Klessig. 1999. Salicylic acid: signal perception and transduction. In: Hooykaas, P.J.J., M.A. Hall, K.R. Libbenga, eds. Biochemistry and molecular biology of plant hormones. Amsterdam: Elsevier Science Publications 513541. doi: 10.1016/S0167-7306(08)60503-7.

Shakirova, F.M., C.R. Allagulova, D.R. Maslennikova, E.O. Klyuchnikova, A.M. Avalbaev, and M.V. Bezrukova. 2016. Salicylic acid-induced protection against cadmium toxicity in wheat plants. Environmental and Experimental Botany 122: 19-28. doi:10.1016/j.envexpbot.2015.08.002.

Shi, G.R., Q.S. Cai, Q.Q. Liu, and L. Wu. 2009. Salicylic acid-mediated alleviation of cadmium toxicity in hemp plants in relation to cadmium uptake, photosynthesis, and antioxidant enzymes. Acta Physiologiae Plantarum 31: 969-977. doi: 10.1007/s11738-009-0312-5.

Shi, Q., and Z. Zhu. 2008. Effects of exogenous salicylic acid on manganese toxicity, element contents and antioxidative system in cucumber. Environmental and Experimental Botany 63: 317-326. doi:10.1016/j.envexpbot.2007.11.003.

Shirasu, K., H. Nakajima, V.K. Rajasekhar, R.A. Dixon, and C. Lamb. 1997. Salicylic acid potentiates an 
agonist-dependent gain control that amplifies pathogen signals in the activation of defense mechanisms. Plant Cell 9: 1-10. doi: 10.1105/tpc.9.2.261. PMID: 9061956.

Sing, I., and K. Shah. 2015. Evidences for suppression of cadmium induced oxidative stress in presence of sulphosalicylic acid in rice seedlings. Plant Growth Regulation 76: 99-110. doi: 10.1007/s10725-015-0023-4.

Singer, A.C., D.E. Crowley, and I.P. Thompson. 2003. Secondary plant metabolites in phytoremediation and biotransformation. Trends Biotechnology 21: 123. doi: 10.1016/S0167-7799 (02)00041-0. PMID: 12628369.

Sinha, S., and S.K. Mukherjee. 2008. Cadmium-induced siderophore production by a high Cd-resistant bacterial strain relieved $\mathrm{Cd}$ toxicity in plants through root colonization. Current Microbiology 56: 55-60. doi: 10.1007/s00284-007-9038-z. PMID: 17899260.

Tao, S., L. Sun, C. Ma, L. Li, G. Li, and L. Hao. 2013. Reducing basal salicylic acid enhances Arabidopsis tolerance to lead or cadmium. Plant and Soil 372: 309-318. doi: 10.1007/s11104-0131749-2.

Tamas, L.., B. Bočová, J. Huttová, L. Liptáková, I. Mistrík, K. Valentovičová, and V. Zelinová 2012. Impact of the auxin signaling inhibitor $p$ chlorophenoxyisobutyric acid on short-term cdinduced hydrogen peroxide production and growth response in barley root tip. Journal of Plant Physiology 169 : 1375-1381. doi: 10.1016/j.jplph.2012.05.023. PMID: 22795748.

Tamas, L., I. Mistrik, A. Alemayehu, V. Zelinova, B. Bocova, and J. Huttova. 2015. Salicylic acid alleviates cadmium-induced stress responses through the inhibition of Cd-induced auxinmediated reactive oxygen species production in barley root tips. Journal of Plant Physiology 173 : 1-8. doi: 10.1016/j.jplph.2014.08.018. PMID: 25462072.

Vlot, A.C., D.M.A. Dempsey, and D.F. Klessig. 2009. Salicylic acid, a multifaceted hormone to combat disease. Annual Review of Phytopathology 47: 177206. doi: 10.1146/annurev.phyto.050908.135202. PMID: 19400653.

Xu, E., and M. Brosché. 2014. Salicylic acid signaling inhibits apoplastic reactive oxygen species signaling. BMC Plant Biology 14:155. doi: 10.1186/1471-2229-14-155. PMID: 24898702.

Xu, L. L., Z.Y. Fan, Y.J. Dong, J. Kong, and X.Y. Bai. 2015. Effects of exogenous salicylic acid and nitric oxide on physiological characteristics of two peanut cultivars under cadmium stress. Biologia Plantarum 59: 171-182. doi: 10.1007/s10535-0140475-9.

Yan, S., and X. Dong. 2014. Perception of the plant immune signal salicylic acid. Current Opinion of Plant Biology 20: 64-68. doi: 10.1016/j.pbi.2014.04.006. PMID: 24840293.

Zawoznik, M., M.D. Groppa, M.L. Tomaro, and M.P. Benavides 2007. Endogenous salicylic acid potentiates cadmium-induced oxidative stress in Arabidopsis thaliana. Plant Science 173: 190-197. doi:10.1016/j.plantsci.2007.05.004.
Zhang, W.N., and W.L. Chen. 2011. Role of salicylic acid in alleviating photochemical damage and autophagic cell death induction of cadmium stress in Arabidopsis thaliana. Photochemical and Photobiological sciences 10: 947-955. doi: 10.1039/c0pp00305k. PMID: 21336371.

Zhang, F., H. Zhang, Y. Xia, G. Wang, L. Xu, and Z. Shen. 2011. Exogenous application of salicylic acid alleviates cadmium toxicity and reduces hydrogen peroxide accumulation in root apoplasts of Phaseolus aureus and Vicia sativa. Plant Cell Reports 30: 1475-1483. doi: 10.1007/s00299-011-1056-4. PMID: 21409549.

Zhang, Y., S. Xu, S. Yang, and Y. Chen. 2015. Salicylic acid alleviates cadmium-induced inhibition of growth and photosynthesis through up-regulating antioxidant defense system in two melon cultivars (Cucumis melo L.). Protoplasma 252: 911-924. doi: 10.1007/s00709-014-0732-y. PMID: 25398649.

Zhao, C.R., T. Ikka, Y. Sawaki, Y. Kobayashi, Y. Suzuki, T. Hibino, S. Sato, N. Sakurai, D. Shibata, and H. Koyama. 2009. Comparative transcriptomic characterization of aluminium, sodium chloride, cadmium and copper rhizotoxicities in Arabidopsis thaliana. BMC Plant Biology 9: 32. doi: 10.1186/1471-2229-9-32. PMID: 19309492. 